\title{
Fully differential decay rate of a standard model Higgs boson into a b-quark pair at NNLO accuracy
}

\author{
Zoltán TRÓCSÁNYI \\ Institute of Physics, University of Debrecen, H-4010 Debrecen P.O.Box 105, Hungary \\ E-mail: zoltan.trocsanyi@cern.ch \\ Gábor SOMOGYI \\ MTA-DE Particle Physics Research Group, Debrecen, Hungary \\ E-mail: gabor.somogyi@cern.ch

\section{Francesco TRAMONTANO} \\ Dipartimento di Fisica, Università di Napoli and INFN, Sezione di Napoli, I-80126 Naples, Italy \\ E-mail: francesco.tramontanodcern.ch
}

We present the application of a general method for computing QCD jet cross sections, called CoLoRFuINNLO, to make predictions for the fully differential decay rate of a standard model Higgs boson into b $\bar{b}$-quark pair at next-to-next-to-leading order accuracy. We also discuss how to model tagged $b$-jets with the flavour- $k_{\perp}$ jet algorithm that is an infrared safe version of the original $k_{\perp}$ algorithm.

The European Physical Society Conference on High Energy Physics

22-29 July 2015

Vienna, Austria

\footnotetext{
* Speaker.

$\dagger$ This research was supported by the Swiss National Science Foundation (grant SCOPES IZ73Z0_152601) and the Hungarian Scientific Research Fund grant K-101482.
} 
Although there is increasing evidence that the boson discovered at the LHC [1,2] is indeed the Higgs-boson of the standard model, the precision of the current measurements still allow that this particle is the first observed member of an extended Higgs sector. Such extended Higgs sectors are more natural from the theoretical point of view. For instance, supersymmetric extensions offer solutions to open questions in the fundamental physical description of Nature, such as the hierarchy problem, or the elementary constituents of cold dark matter.

In order to distinguish whether the observed particle is the standard $(H)$ or one of the supersymmetric neutral Higgs bosons $(\phi)$, it is important to provide accurate predictions for both production and decay of these particles. In this talk we discuss predictions for the $H \rightarrow \mathrm{b} \overline{\mathrm{b}}$ decay mode at the next-to-next-to-leading order (NNLO) accuracy, which can be used to describe the $p p \rightarrow H+X \rightarrow \mathrm{b} \overline{\mathrm{b}}+X$ process at the highest possible accuracy at present, which is NNLO for the production (in the cases when $X$ is unobserved $[3,4]$, a vector boson $[6,5]$, or a jet [7]) and also for the decay $[8,9]$.

In Ref. [9] we presented the first physical application of the CoLoRFuINNLO method [10, 11]. As the width of the Higgs boson is very narrow, the narrow-width approximation can be employed to predict the cross section of the $p p \rightarrow H+X \rightarrow \mathrm{b} \overline{\mathrm{b}}+X$ process. In this approximation the differential distribution for an observable $O_{b \bar{b}}$ (such as the transverse momentum $p_{\perp, b \bar{b}}$ or invariant mass $m_{b \bar{b}}$ of the $b \bar{b}$-jet pair), including up to NNLO corrections for both production and decay is a sum of three contributions:

$$
\begin{aligned}
& \frac{\mathrm{d} \sigma}{\mathrm{d} O_{b \bar{b}}}=\left[\frac{\mathrm{d}^{2} \sigma_{p p \rightarrow H+X}^{(0)}}{\mathrm{d} p_{\perp, H} \mathrm{~d} \eta_{H}} \frac{\mathrm{d} \Gamma_{H \rightarrow \mathrm{b} \overline{\mathrm{b}}}^{(0)} / \mathrm{d} O_{b \bar{b}}+\mathrm{d} \Gamma_{H \rightarrow \mathrm{b} \overline{\mathrm{b}}}^{(1)} / \mathrm{d} O_{b \bar{b}}+\mathrm{d} \Gamma_{H \rightarrow \mathrm{b} \overline{\mathrm{b}}}^{(2)} / \mathrm{d} O_{b \bar{b}}}{\Gamma_{H \rightarrow \mathrm{b} \overline{\mathrm{b}}}^{(0)}+\Gamma_{H \rightarrow \mathrm{b} \overline{\mathrm{b}}}^{(1)}+\Gamma_{H \rightarrow \mathrm{b} \overline{\mathrm{b}}}^{(2)}}\right. \\
& \left.+\frac{\mathrm{d}^{2} \sigma_{p p \rightarrow H+X}^{(1)}}{\mathrm{d} p_{\perp, H} \mathrm{~d} \eta_{H}} \frac{\mathrm{d} \Gamma_{H \rightarrow \mathrm{b} \overline{\mathrm{b}}}^{(0)} / \mathrm{d} O_{b \bar{b}}+\mathrm{d} \Gamma_{H \rightarrow \mathrm{b} \overline{\mathrm{b}}}^{(1)} / \mathrm{d} O_{b \bar{b}}}{\Gamma_{H \rightarrow \mathrm{b} \overline{\mathrm{b}}}^{(0)}+\Gamma_{H \rightarrow \mathrm{b} \overline{\mathrm{b}}}^{(1)}}+\frac{\mathrm{d}^{2} \sigma_{p p \rightarrow H+X}^{(2)}}{\mathrm{d} p_{\perp, H} \mathrm{~d} \eta_{H}} \frac{\mathrm{d} \Gamma_{H \rightarrow \mathrm{b} \overline{\mathrm{b}}}^{(0)} / \mathrm{d} O_{b \bar{b}}}{\Gamma_{H \rightarrow \mathrm{b} \overline{\mathrm{b}}}^{(0)}}\right] \times \mathrm{Br}(H \rightarrow \mathrm{b} \overline{\mathrm{b}}) .
\end{aligned}
$$

Here we concentrate on the second factor in the first term in the brackets.

The physical observables considered in Ref. [9] were flavour blind. Standard jet algorithms, like the $k_{\perp}$ or anti- $k_{\perp}$ algorithms cannot be applied directly to making predictions for flavour-tagged jets, such as the $b$-jets in our example as those are infrared unsafe beyond NLO accuracy [12]. The origin of IR unsafety is the splitting of a gluon into a b-quark pair when the b-quark is considered massless, as in our computation. In the double real emission such a splitting leads to three $b$-jets in the final state (two from the Higgs boson and one from the gluon) if the b-quarks are nearly collinear. In the limit $m_{\mathrm{b}}=0$ the matrix element for this collinear splitting is singular and requires a regularization by subtraction in the CoLoRFuINNLO method. However, the subtraction term contains only two $b$-jets and a gluon jet, therefore its contribution falls into a different bin from that of the double real emission in the singular limit.

There is a conceptually simple way to make IR-safe predictions with massless b-quarks. The flavour- $k_{\perp}$ algorithm of Ref. [12] can be used to define IR safe jet clustering. For the present case, this algorithm can be obtained easily by assigning an additive flavour number +1 to a b-quark and -1 to an anti-b quark. All other partons are considered flavourless with flavour number 0 . Combining two b-quarks by the jet algorithm leads to a pseudo-particle with flavour number 2 , while the combination of a b-quark with an anti-b quark results in a flavourless pseudo-particle. In this way the collinear splitting of a gluon into a $b \bar{b}$-pair in the double real emission contribution 
gives a flavourless jet, just like the corresponding subtraction term does, so the two contributions (double real and the subtraction) fall into the same bin. In this talk we present predictions obtained using the flavour- $k_{\perp}$ algorithm.

The CoLoRFuINNLO method is a general subtraction scheme for computing QCD jet cross sections at the NNLO accuracy. Presently, it has been worked out for colourless initial states and massless partons. In devising the scheme, we set the following requirements useful for efficient numerical implementations and automation:

- the subtractions should be defined and recorded explicitly for all degrees of freedom (momentum, spin, colour and flavour) and for arbitrary processes;

- the method should yield fully differential predictions in four space-time dimensions (so arbitrary detector cuts can be employed and matching to shower Monte Carlo programs is possible);

- the subtractions should be fully local in the multidimensional phase space therefore the correctness of the subtraction terms can be checked in arbitrarily chosen phase-space points;

- the subtractions can be constrained over the phase space (as physical predictions cannot depend on such constraints, this provides an important check of the predictions and a tool for optimization).

In addition to these requirements, the method represents a deeper understanding of the underlying quantum field theory if the coefficients of the poles in singular integrals are computed analytically, which is therefore also considered as a desired feature.

The cross section for $m$-jet production in perturbation theory can be written as $\sigma=\sigma_{\mathrm{LO}}+$ $\sigma_{\mathrm{NLO}}+\sigma_{\mathrm{NNLO}}+\ldots$. The cross section at LO is simply the integral of the fully differential Born cross section of $m$ final-state particles times a function $J_{m}\left(\left\{p_{i}\right\}\right)$, called jet function, which depends on the final-state momenta and defines the $m$-jet physical observable. The fully differential NLO correction is a sum of two contributions:

$$
\mathrm{d} \sigma_{\mathrm{NLO}}=\mathrm{d} \sigma_{m+1}^{\mathrm{R}} J_{m+1}+\mathrm{d} \sigma_{m}^{\mathrm{V}} J_{m}
$$

where the real correction $\mathrm{d} \sigma_{m+1}^{\mathrm{R}} J_{m+1}\left(\left\{p_{i}\right\}\right)$ is essentially the fully differential Born cross section of $m+1$ particles, allowing one particle becoming unresolved (collinear to another one or soft, meaning a particle with vanishing energy) making the $m$-particle and $(m+1)$-particle final states degenerate, while the virtual correction $\mathrm{d}_{m}^{\mathrm{V}} J_{m}\left(\left\{p_{i}\right\}\right)$ is the interference of the one-loop amplitude (first quantum corrections) with the Born amplitude. Both of these terms are separately singular even after ultraviolet renormalization due to integrations over the unresolved momenta (either real, or virtual in the loop), which lead to infrared divergences. However, according to the KinoshitaLee-Nauenberg theorem, their sum is finite if $J$ defines an infrared-safe physical observable.

In constructing general methods one first regulates both real and virtual corrections with dimensional regularization, which amounts to computing the phase space and loop integrals in $d=4-2 \varepsilon$ dimensions. Then the key idea is to devise a suitable approximate cross section $\mathrm{d} \sigma_{m+1}^{\mathrm{A}}$ that has the same point-wise singular behaviour as $\mathrm{d} \sigma_{m+1}^{\mathrm{R}}$ in $d$ dimensions (i.e. for arbitrary $\varepsilon$ ) in all phase-space regions where one particle becomes unresolved (so the subtraction is local). Next we integrate $\mathrm{d} \sigma_{m+1}^{\mathrm{A}}$ over the phase space of the unresolved momentum in $d$ dimensions and sum 
over unresolved discrete degrees of freedom (spin, flavour and colour - all these steps are denoted symbolically by $\int_{1}$ below), of which the jet function that multiplies $\mathrm{d} \sigma_{m+1}^{\mathrm{A}}$ is independent. The result contains $1 / \varepsilon^{2}$ and $1 / \varepsilon$ poles that cancel similar explicit poles in $\mathrm{d} \sigma_{m}^{\mathrm{V}}$. Thus we rewrite Eq. (2) in a reorganized form as a sum of two new contributions:

$$
\begin{gathered}
\sigma_{\mathrm{NLO}}=\int_{m+1} \mathrm{~d} \sigma_{m+1}(\varepsilon)+\int_{m} \mathrm{~d} \sigma_{m}(\varepsilon), \\
\mathrm{d} \sigma_{m+1}(\varepsilon)=\mathrm{d} \sigma_{m+1}^{\mathrm{R}} J_{m+1}-\mathrm{d} \sigma_{m+1}^{\mathrm{R}, \mathrm{A}_{1}} J_{m}, \quad \mathrm{~d} \sigma_{m}(\varepsilon)=\left[\mathrm{d} \sigma_{m}^{\mathrm{V}}+\int_{1} \mathrm{~d} \sigma_{m+1}^{\mathrm{R}, \mathrm{A}_{1}}\right] J_{m}
\end{gathered}
$$

where both $\mathrm{d} \sigma_{m+1}(\varepsilon)$ and $\mathrm{d} \sigma_{m}(\varepsilon)$ are finite and can be integrated in any dimensions. As we are interested in the physical limit $\varepsilon \rightarrow 0$, we can compute $\mathrm{d} \sigma_{m+1}(0)$ and $\mathrm{d} \sigma_{m}(0)$ using Monte Carlo integrations.

The term $\sigma^{\mathrm{NNLO}}$ in the perturbative expansion of the cross section is a sum of three contributions: (i) the double real correction $\mathrm{d} \sigma_{m+2}^{\mathrm{RR}} J_{m+2}\left(\left\{p_{i}\right\}\right)$, which is essentially the fully differential Born cross section of $m+2$ particles, allowing one or two particles becoming unresolved, making either the m-particle or the $(m+1)$-particle final states kinematically degenerate with the $(m+2)$ particle final state; (ii) the real-virtual correction $\mathrm{d} \sigma_{m+1}^{\mathrm{RV}} J_{m+1}\left(\left\{p_{i}\right\}\right)$, which is the interference of the one-loop amplitude (first quantum corrections to the Born one) with the Born amplitude for $(m+1)$-particle final states, containing explicit poles emerging in the loop integral and allowing one particle becoming unresolved; (iii) the double virtual correction $\mathrm{d} \sigma_{m}^{\mathrm{VV}} J_{m}\left(\left\{p_{i}\right\}\right)$ that contains the second quantum (loop) corrections to the amplitude for $m$-particle final states. All three terms are separately divergent even after ultraviolet renormalization due to integrations over the unresolved momenta, which lead to infrared divergences, but their sum is finite if $J$ defines an infraredsafe physical observable. Our goal is to construct a reorganization of the terms into three finite integrals without changing the sum.

In order to find such a reorganization, we use two key concepts that were the basis of general methods for computing cross sections at NLO: (i) the infrared and collinear factorization of QCD matrix elements and (ii) mappings of the phase space which allows for integrating over the unresolved real particles independently of the jet function. In addition we also use a third cornerstone that is new at NNLO: (iii) a process independent way of disentangling overlapping singularities of the amplitudes both among various doubly-unresolved phase-space regions as well as singly- and doubly-unresolved ones.

The infrared factorization of QCD matrix elements has been known for a long time both for soft and for collinear multiple emissions in tree amplitudes $[13,14]$ and in their one-loop versions $[15,16]$. However, points (ii) and (iii) above are highly non-trivial due to the complex, overlapping structure of infrared divergences (for instance when collinear particles become also soft) as shown by the lack of completely satisfactory solutions for NNLO computations. For the third point we provided a solution valid at NNLO in [17], and a general one, valid at any order in perturbation theory in [18]. Once the problem of overlapping singularities is solved, we obtain approximate matrix elements that have the same point-wise singular behaviour as the original matrix element, but only in the strict singular limits. In order to be able to define the approximate cross sections, such as $\mathrm{d} \sigma_{m+1}^{\mathrm{A}}$ NLO case, we also need to introduce mappings of the phase space such that the variables of resolved and unresolved momenta factorize. Mappings that respect the separation of 
the overlapping singularities were presented in $[10,11]$ where the three divergent contributions were transformed into three finite ones, $\sigma^{\mathrm{NNLO}}=\sigma_{m+2}+\sigma_{m+1}+\sigma_{m}$. Each of those terms is finite separately, thus can be computed in four dimensions using Monte Carlo integrations. Symbolically, for the fully differential contributions we have

$$
\begin{gathered}
\mathrm{d} \sigma_{m+2}=\left\{\mathrm{d} \sigma_{m+2}^{\mathrm{RR}} J_{m+2}-\mathrm{d} \sigma_{m+2}^{\mathrm{RR}, \mathrm{A}_{2}} J_{m}-\left[\mathrm{d} \sigma_{m+2}^{\mathrm{RR}, \mathrm{A}_{1}} J_{m+1}-\mathrm{d} \sigma_{m+2}^{\mathrm{RR}, \mathrm{A}_{12}} J_{m}\right]\right\}_{\varepsilon=0}, \\
\mathrm{~d} \sigma_{m+1}=\left\{\left[\mathrm{d} \sigma_{m+1}^{\mathrm{RV}}+\int_{1} \mathrm{~d} \sigma_{m+2}^{\mathrm{RR}, \mathrm{A}_{1}}\right] J_{m+1}-\left[\mathrm{d} \sigma_{m+1}^{\mathrm{RV}, \mathrm{A}_{1}}+\left(\int_{1} \mathrm{~d} \sigma_{m+2}^{\mathrm{RR}, \mathrm{A}_{1}}\right)^{\mathrm{A}_{1}}\right] J_{m}\right\}_{\varepsilon=0}, \\
\mathrm{~d} \sigma_{m}=\left\{\mathrm{d} \sigma_{m}^{\mathrm{VV}}+\int_{2}\left[\mathrm{~d} \sigma_{m+2}^{\mathrm{RR}, \mathrm{A}_{2}}-\mathrm{d} \sigma_{m+2}^{\mathrm{RR}, \mathrm{A}_{12}}\right]+\int_{1}\left[\mathrm{~d} \sigma_{m+1}^{\mathrm{RV}, \mathrm{A}_{1}}+\left(\int_{1} \mathrm{~d} \sigma_{m+2}^{\mathrm{RR}, \mathrm{A}_{1}}\right)^{\mathrm{A}_{1}}\right]\right\}_{\varepsilon=0} J_{m} .
\end{gathered}
$$

The $A_{1}$ terms regularize the one-particle (single) unresolved singularities, the $A_{2}$ term regularizes the double unresolved singularities, while the $A_{12}$ term has two purposes: it regularizes the double unresolved singularities in the RR, $\mathrm{A}_{1}$ subtraction and also the single unresolved ones in the RR, $\mathrm{A}_{2}$ subtraction.

The simultaneous factorization of the phase space and the matrix elements allows for integrating out the unresolved particles. In addition to integrating over momenta, one also has to sum over the spin, colour and flavour degrees of freedom of the unresolved particles (all these steps are denoted symbolically by $\int_{1}$ and $\int_{2}$ where the index shows the number of unresolved particles), which finally leads to integrated subtraction terms that have to be added to cross sections with less particles in the final state (shown with colour code in Eqs. (4) and (6)). Integration of the subtraction terms over the unresolved momenta requires the evaluation of many cumbersome multi-dimensional integrals. These integrals are divergent in $d=4$ dimensions and are regulated by dimensional regularization in $d=4-2 \varepsilon$ dimensions.

For an actual computation we need the Laurent expansion in $\varepsilon$. In $d=4-2 \varepsilon$ dimensions the divergences manifest in $1 / \varepsilon^{n}$ poles $(n=1,2,3,4)$. The coefficients of the poles can be extracted either using sector decomposition [19] or from Mellin-Barnes representations of the integrals [20]. The coefficients appear in the form of parametric integrals that can be computed numerically with high precision [21] or analytically in terms of Goncharov polylogarithms [22] that can be rewritten in terms of ordinary polylogarithms in the cases we have encountered so far. The numerical integration provides an efficient check of the analytic computations as well as a quick method to compute the integrated subtractions at fixed phase-space points. We have implemented both fully numerical and analytical computations of the coefficients of the emerging $1 / \varepsilon^{n}$ poles $(n=1,2,3$, 4) $[23,24,25,26,27]$. These poles must cancel in the $(n+1)$-particle and $n$-particle cross sections against the explicit poles in $\mathrm{d} \sigma_{m+1}^{\mathrm{RV}}$ and $\mathrm{d} \sigma_{m}^{\mathrm{VV}}$. We checked this cancellation analytically for $m=2$ and 3 , which is a highly non-trivial check of the computations.

In our implementation the phase space of the subtractions can be constrained by a dimensionless parameter (a number $\alpha_{0} \in(0,1]$ ). As physical predictions cannot depend on these numbers, checking the independence of the full predictions of $\alpha_{0}$ gives a strong check of correctness. Such a check is shown in Fig. 1.a where the pseudorapidity of the leading jet (measured from an arbitrarily fixed axis in the rest frame of the Higgs boson), clustered with the Durham algorithm [28] at $y_{\text {cut }}=0.05$, can be seen with $\left(\alpha_{0}=0.1\right)$ and without $\left(\alpha_{0}=1\right)$ phase-space restriction. We obtained these predictions using a fixed renormalization scale $\mu_{\mathrm{R}}=\mu_{0}=m_{H}$. Clearly, the predictions are 

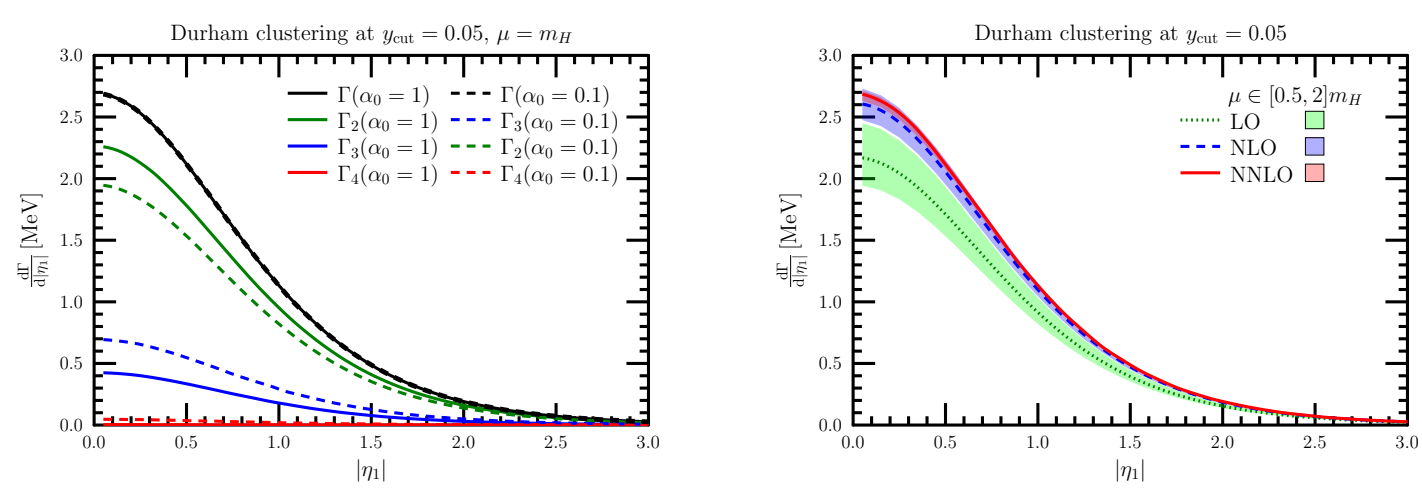

Figure 1: Rapidity distribution of the hardest jet: (a) dependence of the various contributions on $\alpha_{0}$, (b) dependence of the physical prediction on the renormalization scale. The bands represent the variation of the scale at LO, NLO and NNLO around the default one in the range $\left[\mu_{0} / 2,2 \mu_{0}\right]$.

independent of $\alpha_{0}$, so it can be tuned for better numerical efficiency of the complete computation. For instance, the same numerical accuracy for the final NNLO prediction can be achieved with $\alpha_{0}=0.1$ as with $\alpha_{0}=1$ with 2.5 times more CPU time needed for the latter. This is due to the reduced number of subtractions needed to compute at each phase space point, which is 52 for $\alpha_{0}=1$, while only 14.5 on average for $\alpha_{0}=0.1$. The scale dependence is exhibited in Fig. 1.b as bands corresponding to the scale variation around the default one in the range $\left[\mu_{0} / 2,2 \mu_{0}\right]$, obtained with $\alpha_{0}=1$. While the NLO band is clearly above the LO one over almost the whole rapidity range, the NNLO band is inside the NLO one but significantly narrower, which shows nice convergence of the perturbative prediction at this order.

Fig. 2 shows the distributions of the transverse momenta of the two hardest $b$-jets (ordered according to their energies) in the rest-frame of the Higgs boson when jets are clustered using the flavour- $k_{\perp}$ algorithm at $y_{\text {cut }}=0.05$. For the leading $b$-jet the NLO prediction is above the LO band even more significantly as in Fig. 1b. The scale dependence is reduced significantly at NNLO and the band lies within the NLO one. For the sub-leading $b$-jet the distribution at LO coincides with that of the leading one because there are only two back-to-back b-quarks in the final state. At NLO the dominant contribution is the three-parton final state for which the prediction is leading order. The extra parton softens the spectrum. The NNLO band again shows significant stabilization of the prediction.

We presented predictions for the $H \rightarrow \mathrm{b} \overline{\mathrm{b}}$ decay mode at the NNLO accuracy. For tagged $b$-jets the flavour- $k_{\perp}$ algorithm is infrared safe beyond NLO. We found significant reduction of the remaining theoretical uncertainties and stable predictions at NNLO.

\section{References}

[1] G. Aad et al. [ATLAS Collaboration], Phys. Lett. B 710, 49 (2012) [arXiv:1202.1408 [hep-ex]].

[2] S. Chatrchyan et al. [CMS Collaboration], Phys. Lett. B 710, 26 (2012) [arXiv:1202.1488 [hep-ex]].

[3] C. Anastasiou, K. Melnikov and F. Petriello, Phys. Rev. Lett. 93, 262002 (2004) [hep-ph/0409088].

[4] S. Catani and M. Grazzini, Phys. Rev. Lett. 98, 222002 (2007) [hep-ph/0703012]. 

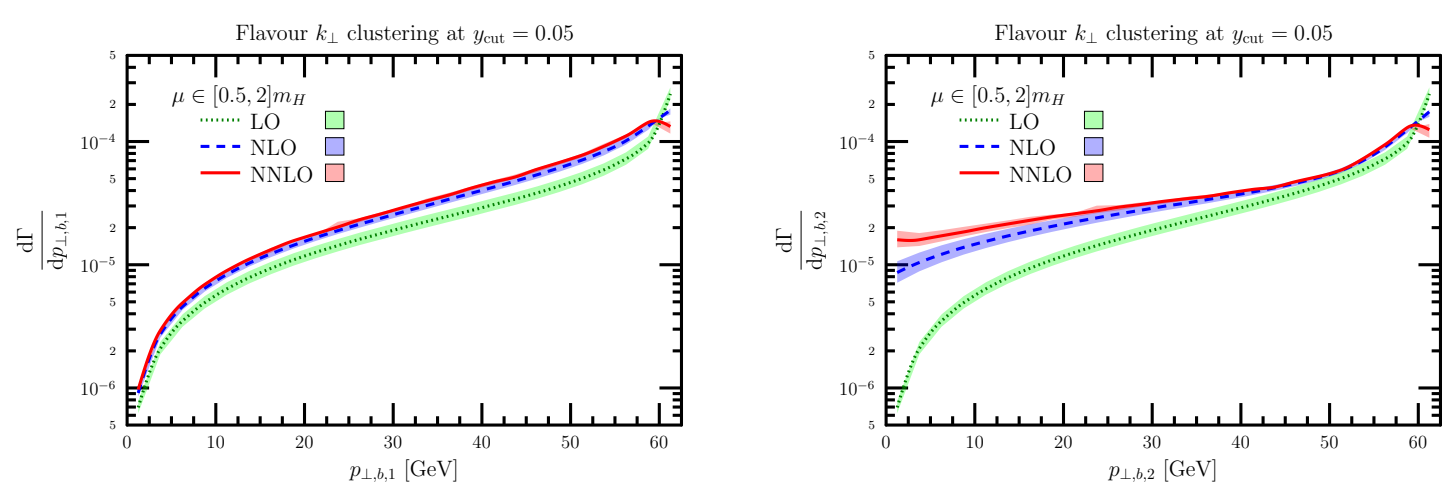

Figure 2: Distributions of the transverse momenta for (a) the leading $b$-jet, (b) the sub-leading $b$-jet, with jets ordered according to their energies. The bands represent the variation of the scale at LO, NLO and NNLO around the default one in the range $\left[\mu_{0} / 2,2 \mu_{0}\right]$.

[5] G. Ferrera, M. Grazzini and F. Tramontano, Phys. Lett. B 740, 51 (2015) [arXiv:1407.4747 [hep-ph]].

[6] G. Ferrera, M. Grazzini and F. Tramontano, JHEP 1404, 039 (2014) [arXiv:1312.1669 [hep-ph]].

[7] R. Boughezal, F. Caola, K. Melnikov, F. Petriello and M. Schulze, Phys. Rev. Lett. 115, no. 8, 082003 (2015) [arXiv:1504.07922 [hep-ph]].

[8] C. Anastasiou, F. Herzog and A. Lazopoulos, JHEP 1203, 035 (2012) [arXiv:1110.2368 [hep-ph]].

[9] V. Del Duca, C. Duhr, G. Somogyi, F. Tramontano and Z. Trócsányi, JHEP 1504, 036 (2015) [arXiv:1501.07226 [hep-ph]].

[10] G. Somogyi, Z. Trócsányi and V. Del Duca, JHEP 0701, 070 (2007) [hep-ph/0609042].

[11] G. Somogyi and Z. Trócsányi, JHEP 0701, 052 (2007) [hep-ph/0609043].

[12] A. Banfi, G. P. Salam and G. Zanderighi, Eur. Phys. J. C 47, 113 (2006) [hep-ph/0601139].

[13] J. M. Campbell and E. W. N. Glover, Nucl. Phys. B 527, 264 (1998) [hep-ph/9710255].

[14] S. Catani and M. Grazzini, Nucl. Phys. B 570, 287 (2000) [hep-ph/9908523].

[15] Z. Bern, V. Del Duca, W. B. Kilgore and C. R. Schmidt, Phys. Rev. D 60, 116001 (1999) [hep-ph/9903516].

[16] S. Catani and M. Grazzini, Nucl. Phys. B 591, 435 (2000) [hep-ph/0007142].

[17] G. Somogyi, Z. Trócsányi and V. Del Duca, JHEP 0506, 024 (2005) [hep-ph/0502226].

[18] Z. Nagy, G. Somogyi and Z. Trócsányi, hep-ph/0702273 [HEP-PH].

[19] T. Binoth and G. Heinrich, Nucl. Phys. B 693, 134 (2004) [hep-ph/0402265].

[20] G. Somogyi, JHEP 1304, 010 (2013) [arXiv:1301.3919 [hep-ph]].

[21] S. Borowka, J. Carter and G. Heinrich, Comput. Phys. Commun. 184, 396 (2013) [arXiv:1204.4152 [hep-ph]].

[22] A. B. Goncharov. Math. Research Letters. 5(4), 497 (1998).

[23] G. Somogyi and Z. Trócsányi, JHEP 0808, 042 (2008) [arXiv:0807.0509 [hep-ph]].

[24] U. Aglietti, V. Del Duca, C. Duhr, G. Somogyi and Z. Trócsányi, JHEP 0809, 107 (2008) [arXiv:0807.0514 [hep-ph]].

[25] P. Bolzoni, S. O. Moch, G. Somogyi and Z. Trócsányi, JHEP 0908, 079 (2009) [arXiv:0905.4390 [hep-ph]].

[26] P. Bolzoni, G. Somogyi and Z. Trócsányi, JHEP 1101, 059 (2011) [arXiv:1011.1909 [hep-ph]].

[27] V. Del Duca, G. Somogyi and Z. Trócsányi, JHEP 1306, 079 (2013) [arXiv:1301.3504 [hep-ph]].

[28] S. Catani, Y. L. Dokshitzer, M. Olsson, G. Turnock and B. R. Webber, Phys. Lett. B 269, 432 (1991). 